\title{
Scintigraphic study of relation between left ventricular peak systolic pressure and end-systolic volume in patients with coronary artery disease and normal subjects
}

\author{
JOHN WATKINS, ^ ROBERT SLUTSKY, JULIO TUBAU, JOEL KARLINER \\ From the Division of Cardiology, University of Califormia, San Diego, Califormia, USA
}

SUMMARY We have studied the relation between left ventricular peak systolic pressure and endsystolic volume in 12 normal subjects and 12 patients with coronary artery disease. Peak systolic pressure was measured with a cuff sphygmomanometer and left ventricular volumes were determined by radionuclide ventriculography.

Observations were made at rest, after $1 \mathrm{mg}$ atropine, and during at least three increments of an infusion of phenylephrine which raised systolic blood pressure by $45 \mathrm{mmHg}$ while heart rate was held constant.

The relation between peak systolic pressure and end-systolic volume at a constant heart rate was found to be linear in all subjects. The slope of this line was steeper in normal subjects than in patients with coronary artery disease and correlated exponentially with the ejection fraction determined simultaneously. The end-systolic volume extrapolated to zero pressure $\left(V_{0}\right)$ was not significantly different between the two groups and correlated poorly with the ejection fraction.

The simple ratio of peak systolic pressure to end-systolic volume, at rest and after drug interventions, was of similar sensitivity to the ejection fraction in the detection of patients with left ventricular dysfunction caused by coronary artery disease. In normal subjects, however, this ratio rose after atropine whereas the ejection fraction was unchanged.

The apparent superiority of the ratio of peak systolic pressure to end-systolic volume over the ejection fraction in the detection of the positive inotropic response to increasing heart rate, coupled with the linearity of this relation over a wide range of systolic blood pressure, suggests that the systolic pressure-volume relation will prove useful in the evaluation of cardiac drug effects in man.

None of the indices derived from the systolic pressure volume relation, however, was more sensitive than the ejection fraction derived simultaneously, in the detection of patients with left ventricular dysfunction resulting from coronary artery disease.

The relation between left ventricular pressure and volume at end-systole in experimental models has been shown to be a sensitive and relatively preloadindependent index of the prevailing inotropic state. ${ }^{1-3}$

The utility of this relation as a putative index of inotropic state in man has been enhanced by the

This study was supported by an NIH grant.

^Harkness Fellow Of The Commonwealth Fund Of New York.

Accepted for publication 23 March 1982 finding that peak systolic pressure can be substituted for end-systolic pressure without significantly altering these properties. ${ }^{4-6}$

Considerable interest has thus been shown in this relation, both as a method of studying cardiac drug effects and also as a means of detecting myocardial dysfunction caused by coronary artery disease. ${ }^{4-10}$

The simple ratio of peak systolic pressure to endsystolic volume ( $\mathrm{P} / \mathrm{V}$ ratio), the slope of this relation over a physiological range of blood pressure (P/V slope), and the theoretical value of end-systolic volume extrapolated to zero systolic pressure $\left(V_{0}\right)$ have 
all been used to represent the end-systolic pressurevolume relation, but none has been widely evaluated in man.

We have therefore undertaken a scintigraphic study in normal subjects and patients with coronary artery disease: (i) to confirm the linearity of the peak systolic pressure-end-systolic volume relation in man by a method that does depend on geometric assumptions about ventricular morphology at end-systole; (ii) to compare the $\mathrm{P} / \mathrm{V}$ ratio, the $\mathrm{P} / \mathrm{V}$ slope, and $\mathrm{V}_{0}$-all derived at a constant inotropic state-with the ejection fraction obtained simultaneously; (iii) to compare the sensitivity of these indices in the detection of impaired myocardial function resulting from coronary artery disease.

\section{Subjects and methods}

The study was undertaken in 24 normotensive male volunteers. Twelve subjects (mean age 51, range 41 to 69 years) had sustained a myocardial infarction at least six months previously, as evidenced by a typical history of chest pain, raised serum creatine kinase levels, and characteristic electrocardiographic changes (for complete clinical details, see Table 1). No patient had clinical evidence of valvular heart disease or peripheral vascular disease and none had taken any drugs except diuretics within $\mathbf{4 8}$ hours of the study.

The other 12 subjects were normal (mean age 34, range 19 to 58 years) with no clinical evidence of cardiovascular disease. They were taking no drugs and had no abnormalities on resting or exercise electrocardiograms. Written informed consent was obtained from all subjects and the procedure was approved by the Human Subjects Committee of the University of California, San Diego. Neither smoking nor the consumption of caffeine-containing beverages was permitted for four hours before the study.

On arrival at the laboratory, subjects were instructed to lie supine and to remain still throughout the study. A 19 gauge "butterfly" needle (Abbott Ltd) was inserted into a vein in the right arm for administration of drugs. An 18 gauge intravenous cannula (Jelco Ltd) was introduced into a forearm vein on the left arm for blood sampling.

\section{MEASUREMENTS}

Standard lead II of a surface electrocardiogram was continuously displayed on a cardiac monitor (Hewlett Packard Ltd). A precise heart rate was obtained at each phase in the study from the number of cardiac cycles recorded by the gamma camera during a five minute electrocardiogram gated left ventricular scintigram (see later).

Blood pressure, taken by the same investigator, was measured by auscultation in duplicate from the right arm every 60 seconds during left ventricular scintigraphy, using a standard mercury sphygmomanometer (Baumanometer Ltd). The systolic blood pressure used in the determination of each point in the systolic pressure/volume relation was thus the mean of 10 readings taken over a five minute period. (Blood pressure was also measured in the left arm at the beginning of the study and disparities between the two arms of $>15 \mathrm{mmHg}$ were not found.)

Left ventricular ejection fraction, end-systolic volume, and end-diastolic volume were determined by equilibrium radionuclide ventriculography (see data analysis).

The patient's own red cells were labelled with $99 m$ technetium (Tc) using the method of Smith and Richards. ${ }^{11}$ Briefly, $4 \mathrm{ml}$ venous blood were drawn into a heparinised syringe and incubated for five minutes with freeze-dried stannous citrate in a sterile

Table 1 Clinical details of patients with previous myocardial infarction: symptomatic details and clinical class refer to time of study

\begin{tabular}{|c|c|c|c|c|c|c|c|}
\hline Subject & $\begin{array}{l}\text { Location of } \\
\text { myocardial } \\
\text { infarction }\end{array}$ & $\begin{array}{l}\text { Coromary } \\
\text { bypass } \\
\text { surgery }\end{array}$ & $\begin{array}{l}\text { Congestive } \\
\text { heart failure }\end{array}$ & Angina & Arrhythimias & $\begin{array}{l}\text { NYHA } \\
\text { clinical } \\
\text { class }\end{array}$ & Medications \\
\hline $\mathrm{HC}$ & Anterior & - & - & + & - & I & Nitrates \\
\hline $\mathbf{K B}$ & Inferior & - & - & + & - & I & Nitrates \\
\hline FC & Inferior & - & - & + & + & II & $\begin{array}{l}\text { Frusemide, } \\
\text { nitrates, } \\
\text { propranolol }\end{array}$ \\
\hline RS & Anterior & - & + & + & - & II & $\begin{array}{l}\text { Quinidine, } \\
\text { digoxin, } \\
\text { nitrates }\end{array}$ \\
\hline CD & Subendocardial & - & - & - & + & I & Quinidine \\
\hline MW & Inferior & + & - & - & - & I & Digoxin \\
\hline $\mathbf{A H}$ & Anterior & - & - & - & - & I & - \\
\hline $\mathbf{M R}$ & Anterior & - & - & - & - & I & \\
\hline LG & Inferior & - & - & + & - & I & Nitrates \\
\hline WW & Inferior & + & - & - & - & I & \\
\hline GM & Inferior & - & - & - & + & I & $\begin{array}{l}\text { Quinidine, } \\
\text { nitrates }\end{array}$ \\
\hline HP & Anterior & + & - & - & - & I & - \\
\hline
\end{tabular}


evacuated glass tube. The tube was then centrifuged upside down for five minutes at $1300 \times g$ and the red cells carefully removed by needle aspiration through the rubber stopper.

Finally, the red cells were incubated in a sterile tube containing ${ }^{99 \mathrm{~m}} \mathrm{Tc}$ (Nuclear Pharmacy Ltd) for a further five minutes before $1 \mathrm{ml}$ red cell suspension, containing 15 to $20 \mathrm{mCi}{ }^{99} \mathrm{~m} \mathrm{Tc}$, was reinjected intravenously to effect the blood pool label. Great care was taken to ensure a sterile technique and also to minimise damage to the red cells during the labelling procedure.

A single crystal gamma camera (Picker Dyna-Mo) fitted with a parallel hole, general purpose collimator, was then positioned over the subject's chest wall in a $45^{\circ}$ left anterior oblique position, with 5 to $10^{\circ}$ caudal tilt. The camera position was then further adjusted if necessary so that the image of the left ventricle was clearly separated from that of the right ventricle and the left atrium. Thereafter, the camera position remained unchanged.

\section{PROCEDURE}

After the subjects were in a basal haemodynamic state, a five minute equilibrium left ventricular scintigram, gated from a modified V5 electrocardiographic lead, was performed. Blood pressure was measured in duplicate each minute. At the mid-point of the scintigraphic acquisition, a $10 \mathrm{ml}$ venous sample was drawn, from which $4 \mathrm{ml}$ was pipetted for later gamma counting.

Then, $1 \mathrm{mg}$ atropine sulphate (Abbott Ltd) was injected intravenously over five minutes. This was done to minimise the baroreflex mediated bradycardia that accompanies the infusion of pressor agents such as phenylephrine. After a further 10 minutes, left ventricular scintigraphy, blood sampling, and blood pressure measurements were repeated.

Next, an $80 \mu \mathrm{g} / \mathrm{ml}$ solution of phenylephrine hydrochloride (Winthrop Ltd) in normal saline was infused by means of a variable delivery rate pump (Ivac Ltd). The infusion was started at $80 \mu \mathrm{g} /$ minute and titrated to produce target increments in systolic blood pressure of 15,30 , and $45 \mathrm{mmHg}$. When each target level of systolic blood pressure had been reached, it was held stable for five minutes while left ventricular scintigraphy was repeated. The heart rate was maintained within six beats per minute of that achieved after atropine by supplementary intravenous injections of atropine $(0.1$ to $0.3 \mathrm{mg})$ when necessary. In four patients with coronary artery disease, only two increments of phenylephrine were used because of the initial low ejection fraction.

DATA ANALYSIS

Equilibrium radionuclide studies were processed using a "Muga Cart" on-line computer (Medical Data Systems Ltd). Data recorded from each cardiac cycle during the five minutes of acquisition were divided into 28 equal time frames and integrated to construct a composite cardiac cycle. A rectangular region of interest was then placed around the composite left ventricular image, and an edge detection programme (Mug-O) used to define the boundary of the left ventricle throughout the cardiac cycle. (The edge detection algorithm utilises both absolute count rates and second derivatives of the count rate change in each time frame.) Background counts were automatically subtracted from the left ventricular region of interest to correct for non-cardiac activity. The five minute count rate exceeded 5000 in the left ventricular region of interest at end-diastole in all studies. Thus, a background corrected time-activity curve from the left ventricle was obtained, from which ejection fraction was calculated using the following equation:

Ejection fraction $=$

$\frac{\text { (end-diastolic counts)-(end-systolic counts) }}{\text { (end-diastolic counts) }}$

Left ventricular end-diastolic volume was calculated by the method of Slutsky et al. ${ }^{12}$ Left ventricular counts at end-diastole, corrected for frame time and the number of cardiac cycles processed, were divided by counts from whole blood drawn at the mid-point of each study, corrected for background activity, elapsed time, and radioactive decay.

Thus:

$$
\begin{aligned}
& {\left[\frac{\frac{(\text { end-diastolic counts) }}{\text { cycles processed }} \times \frac{0.04}{\text { time/frame }}}{\left(\begin{array}{c}
\text { Whole blood-Background } \\
\text { counts }
\end{array}\right) \times \begin{array}{c}
\mathrm{e} \cdot 115 \mathrm{x} \text { hours } \\
\text { elapsed }
\end{array}}\right] \times 10^{3}} \\
& =\text { Arbitrary end-diastolic volume units. }
\end{aligned}
$$

Using a least squares linear regression derived from previous comparisons between radionuclide and contrast ventriculography at this institution, ${ }^{13}$ enddiastolic volume was calculated as:

$$
\mathrm{EDV}=\frac{\left(\begin{array}{c}
\text { Arbitrary end-diastolic } \\
\text { volume units }
\end{array}\right)-0.53}{0.51} \mathrm{ml}
$$

Using this determination of end-diastolic volume, stroke volume (SV) was calculated as:

$$
\mathrm{SV}=\mathrm{EF} \times \mathrm{EDV} \mathrm{ml}
$$

and, finally, ESV was calculated as:

$$
\mathrm{ESV}=\mathrm{EDV}-\mathrm{SV} \mathrm{ml}
$$

All ventricular volumes were reported as volume indices, that is $\mathrm{ml} / \mathrm{m}^{2}$ body surface area. The reproducibility of this technique has been validatd in our own and other laboratories. The reproducibility over time of end-diastolic volume and ejection fraction 
using this method was $\pm 7.5 \mathrm{ml}$ and $\pm 3 \%$, respectively. ${ }^{14} 15$ Inter- and intra- observer variability have both been found to be approximately $4 \% .^{15}$

Individual plots were then constructed using three $(n=8)$, four $(n=12)$, or five $(n=4)$ points to define the relation between the mean value of systolic blood pressure during a five minute scintigram and the calculated end-systolic volume index, over a physiological range of blood pressure.

Data were used only if heart rates during the various steps of phenylephrine infusion were successfully maintained within six beats a minute of the initial post-atropine value. Thus, the resting (pre-atropine) data were not included in these plots.

\section{STATISTICAL ANALYSIS}

Statistical comparisons within groups were performed using Wilcoxon's signed rank test and between groups using Wilcoxon's rank sum test. Regression analysis of paired data was performed on a Hewlett Packard model 9830A computer using a program which generated equations for the lines which best described these relations. All data are presented as the mean \pm 1 standard deviation from the mean, except where otherwise stated.

\section{Results}

(1) HAEMODYNAMIC CHANGES AND COMPARATIVE EFFECTS ON P/V RATIO AND EJECTION FRACTION

\section{At rest}

The haemodynamic status of the 12 normal subjects and the 12 patients with coronary artery disease is shown in Table 2. Blood pressure and heart rate were similar in the two groups, the major difference being a larger end-systolic volume in the patients with coronary disease $\left(50 \pm 24 \mathrm{ml} / \mathrm{m}^{2}\right.$ compared with $22 \pm 7$ $\mathrm{ml} / \mathrm{m}^{2}$ in the normals, $\mathrm{p}<0.01$ ).

While the mean $P / V$ ratio was significantly lower in the patients with coronary disease than the normal subjects $\left(2.79 \pm 1.00 \mathrm{mmHg} / \mathrm{ml}\right.$ per $\mathrm{m}^{2}$ compared with $6.23 \pm 2.47 \mathrm{mmHg} / \mathrm{ml} \mathrm{per} \mathrm{m}^{2}, \mathrm{p}<0.01$ ), it was not significantly better than the ejection fraction in the identification of individual patients with left ventricular dysfunction caused by coronary artery disease (Fig. 1).

\section{After atropine}

The response to $1 \mathrm{mg}$ intravenous atropine is shown in the left hand panels of Tables 3 and 4 for normal subjects and patients with coronary artery disease, respectively. Heart rate rose by 20 to 25 beats a minute and diastolic blood pressure rose by 5 to 8 $\mathrm{mmHg}$ in each group. The $\mathrm{P} / \mathrm{V}$ ratio was significantly increased by atropine in the normal subjects (from $6.23 \pm 2.47$ to $7.28 \pm 2.7 \mathrm{mmHg} / \mathrm{ml}$ per $\mathrm{m}^{2}, \mathrm{p}<0.01$ ) but did not rise significantly in the patients with coronary artery disease (from $2.79 \pm 1.00$ to $3.28 \pm 1.66$ $\mathrm{mmHg} / \mathrm{ml}^{\text {per }} \mathrm{m}^{2}$ ).

By contrast, the ejection fraction showed a nonsignificant decline after atropine in both groups. In the patients with coronary disease, stroke volume fell significantly (from $50 \pm 24$ to $32 \pm 12 \mathrm{ml} / \mathrm{m}^{2}, \mathrm{p}<0.01$ ) but was maintained in the control group $(53 \pm 13$ $\mathrm{ml} / \mathrm{m}^{2}$ at rest, $46 \pm 18 \mathrm{ml} / \mathrm{m}^{2}$ after atropine $-\mathrm{NS}$ ).

Fig. 2 is a plot of the relation between the $P / V$ ratio and the ejection fraction after atropine administration. The relation is best described by the exponential equation $y=0.82 \times e^{0.3 X}$, as illustrated. Note that most of the normal subjects lie on the steep part of the curve, where ejection fraction is relatively insensitive to changes in the $\mathrm{P} / \mathrm{V}$ ratio.

Table 2 Resting haemodynamic data in normal subjects and patients with coronary disease

\begin{tabular}{|c|c|c|c|c|c|c|c|c|c|c|c|c|c|c|c|}
\hline \multicolumn{8}{|c|}{ Normal subjects } & \multicolumn{8}{|c|}{ Coronary disease patients } \\
\hline Subject & $\begin{array}{l}B P \\
(m m H g)\end{array}$ & $\begin{array}{l}H R \\
(b p m)\end{array}$ & $\begin{array}{l}E D V I \\
\left(m l / m^{2}\right)\end{array}$ & $\begin{array}{l}E S V I \\
\left(\mathrm{ml} / \mathrm{m}^{2}\right)\end{array}$ & $\begin{array}{l}S V I \\
\left(m l / m^{2}\right)\end{array}$ & $\begin{array}{l}P N \\
(m m H g / m l \\
\left.\text { per } m^{2}\right)\end{array}$ & $\begin{array}{l}E F \\
(\%)\end{array}$ & Subject & $\begin{array}{l}B P \\
(m m H g)\end{array}$ & $\begin{array}{l}H R \\
(b p m)\end{array}$ & $\begin{array}{l}E D V I \\
\left(m l / m^{2}\right)\end{array}$ & $\begin{array}{l}E S V I \\
\left(m l / m^{2}\right)\end{array}$ & $\begin{array}{l}S V I \\
\left(m l / m^{2}\right)\end{array}$ & $\begin{array}{l}P N \\
\underset{\left.\text { per } m^{2}\right)}{ }\end{array}$ & $\begin{array}{l}E F \\
(\%)\end{array}$ \\
\hline $\begin{array}{l}\text { WC } \\
\text { DJ } \\
\text { ML } \\
\text { RC } \\
\text { MB } \\
\text { GM } \\
\text { PW } \\
\text { DE } \\
\text { WE } \\
\text { WM } \\
\text { SS } \\
\text { HR }\end{array}$ & $\begin{array}{l}117 / 69 \\
112 / 61 \\
117 / 71 \\
120 / 70 \\
120 / 79 \\
128 / 70 \\
127 / 79 \\
118 / 75 \\
128 / 69 \\
110 / 82 \\
130 / 75 \\
127 / 74\end{array}$ & $\begin{array}{ll}9 & 67 \\
1 & 71 \\
1 & 58 \\
0 & 70 \\
9 & 54 \\
0 & 58 \\
9 & 52 \\
5 & 72 \\
9 & \mathbf{6 8} \\
2 & 93 \\
5 & 71 \\
4 & \mathbf{8 2}\end{array}$ & $\begin{array}{l}84 \\
90 \\
99 \\
75 \\
72 \\
62 \\
92 \\
50 \\
58 \\
56 \\
72 \\
85\end{array}$ & $\begin{array}{l}23 \\
24 \\
36 \\
22 \\
21 \\
15 \\
26 \\
11 \\
24 \\
26 \\
12 \\
21\end{array}$ & $\begin{array}{l}61 \\
66 \\
63 \\
53 \\
51 \\
47 \\
66 \\
39 \\
34 \\
30 \\
60 \\
64\end{array}$ & $\begin{array}{r}5.09 \\
4.67 \\
3.25 \\
5.45 \\
5.71 \\
8.53 \\
4.88 \\
10.73 \\
5.33 \\
4.23 \\
10.83 \\
6.05\end{array}$ & $\begin{array}{l}73 \\
73 \\
64 \\
70 \\
71 \\
76 \\
71 \\
78 \\
59 \\
53 \\
83 \\
76\end{array}$ & $\begin{array}{l}\text { HC } \\
\text { KB } \\
\text { FC } \\
\text { RS } \\
\text { CD } \\
\text { MW } \\
\text { AH } \\
\text { MR } \\
\text { LG } \\
\text { WW } \\
\text { GM } \\
\text { HP }\end{array}$ & $\begin{array}{l}106 / 65 \\
136 / 68 \\
100 / 65 \\
105 / 70 \\
108 / 62 \\
122 / 84 \\
132 / 80 \\
117 / 60 \\
122 / 71 \\
135 / 75 \\
114 / 72 \\
115 / 75\end{array}$ & $\begin{array}{l}61 \\
80 \\
46 \\
56 \\
88 \\
70 \\
55 \\
71 \\
54 \\
53 \\
53 \\
97\end{array}$ & $\begin{array}{r}81 \\
89 \\
68 \\
121 \\
42 \\
77 \\
68 \\
96 \\
94 \\
129 \\
76 \\
126\end{array}$ & $\begin{array}{l}45 \\
45 \\
24 \\
94 \\
29 \\
40 \\
42 \\
56 \\
32 \\
61 \\
32 \\
98\end{array}$ & $\begin{array}{l}36 \\
44 \\
44 \\
27 \\
13 \\
37 \\
26 \\
40 \\
62 \\
68 \\
44 \\
28\end{array}$ & $\begin{array}{l}2.36 \\
3.02 \\
4.17 \\
1.12 \\
3.72 \\
3.05 \\
3.14 \\
2.09 \\
3.81 \\
2.21 \\
3.56 \\
1.17\end{array}$ & $\begin{array}{l}44 \\
49 \\
65 \\
22 \\
30 \\
48 \\
39 \\
41 \\
66 \\
53 \\
58 \\
22\end{array}$ \\
\hline $\begin{array}{l}M \\
\text { SD }\end{array}$ & $\begin{array}{r}121 / 73 \\
\pm \quad 7 / 6\end{array}$ & $\begin{array}{r}3 \quad 68 \\
\pm 12\end{array}$ & $\begin{array}{r}75 \\
\pm 16\end{array}$ & $\begin{array}{r}22 \\
\pm 7\end{array}$ & $\begin{array}{r}53 \\
\pm 13\end{array}$ & $\begin{array}{r}6.23 \\
\pm \quad 2.47\end{array}$ & $\begin{array}{r}71 \\
\pm 8\end{array}$ & $\begin{array}{l}M \\
\text { SD }\end{array}$ & $\begin{array}{r}118 / 71 \\
+\quad 12 / 7\end{array}$ & $\begin{array}{r}65 \\
\pm 16\end{array}$ & $\begin{array}{r}89 \\
+\quad 26\end{array}$ & $\begin{array}{r}50 \\
+24\end{array}$ & $\begin{array}{r}39 \\
\pm 15\end{array}$ & $\begin{array}{r}2.79 \\
\pm 1.00\end{array}$ & $\begin{array}{r}45 \\
\pm 15\end{array}$ \\
\hline
\end{tabular}

BP, blood pressure; HR, heart rate; EDVI, end-diastolic volume index; ESVI, end-systolic volume index; SVI, stroke volume index; P/V, peak systolic blood pressure/end-systolic volume index ratio; EF, ejection fraction. 


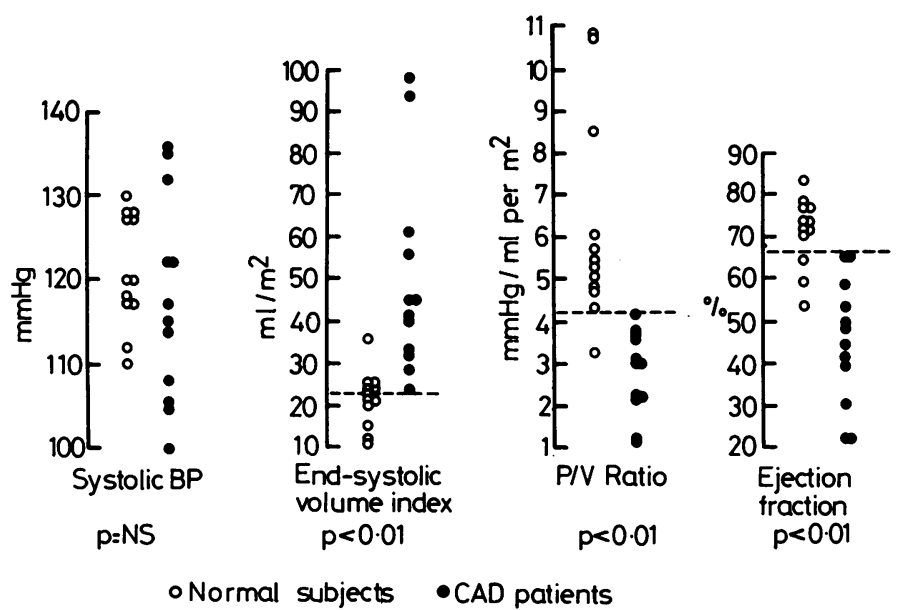

Fig. 1 Comparison of resting values of systolic blood pressure, end-systolic volume, $P N$ ratio, and ejection fraction in normal subjects and patients with coronary artery disease (CAD). The dotted lines denote the values that best discriminate between the two groups.

\section{During phenylephrine}

The maximum haemodynamic respone to the pressor effects of phenylephrine are shown in the right hand panels of Tables 3 and 4 for normal subjects and patients with coronary disease, respectively. Mean systolic blood pressure was similarly raised (42 and 39 $\mathrm{mmHg}$ ) in both groups, and in no subject was heart rate permitted to fall by $>6$ beats/minute from the post-atropine value. The $\mathrm{P} / \mathrm{V}$ ratio fell in both groups but was only significantly reduced in the normal subjects (from $7 \cdot 28 \pm 2.27$ to $5.87 \pm 1.40 \mathrm{mmHg} / \mathrm{ml}$ per $\left.\mathrm{m}^{2}, \mathrm{p}<0.01\right)$.

Mean ejection fraction also fell in both groups during phenylephrine though again this was only

Table 3 Haemodynamic data in normal subjects (left) 10 minutes after $1 \mathrm{mg}$ atropine given intravenously and (right) during maximum infusion rate of phenylephrine

\begin{tabular}{|c|c|c|c|c|c|c|c|c|c|c|c|c|c|c|}
\hline \multicolumn{8}{|c|}{ After atropine } & \multicolumn{7}{|c|}{ During maximum phenylephrine infusion } \\
\hline Subject & $\begin{array}{l}B P \\
(\operatorname{mm} H g)\end{array}$ & $\begin{array}{l}H R \\
(b p m)\end{array}$ & $\begin{array}{l}E D V I \\
\left(m l / m^{2}\right)\end{array}$ & $\begin{array}{l}E S V I \\
\left(m l / m^{2}\right)\end{array}$ & $\begin{array}{l}S V I \\
\left(m l / m^{2}\right)\end{array}$ & $\begin{array}{l}P / V \\
(m m H g / m l \\
\left.\text { per } m^{2}\right)\end{array}$ & $\begin{array}{l}E F \\
(\%)\end{array}$ & $\begin{array}{l}B P \\
(m m H g)\end{array}$ & $\begin{array}{l}H R \\
(b p m)\end{array}$ & $\begin{array}{l}E D V I \\
\left(m l / m^{2}\right)\end{array}$ & $\begin{array}{l}E S V I \\
\left(m l / m^{2}\right)\end{array}$ & $\begin{array}{l}S V I \\
\left(m l / m^{2}\right)\end{array}$ & $\begin{array}{l}P / V \\
(m m H g / m l \\
\left.\text { per } m^{2}\right)\end{array}$ & $\begin{array}{l}E F \\
(\%)\end{array}$ \\
\hline $\begin{array}{l}\text { WC } \\
\text { DJ } \\
\text { ML } \\
\text { RC } \\
\text { MB } \\
\text { GM } \\
\text { PW } \\
\text { DE } \\
\text { WE } \\
\text { WM } \\
\text { SS } \\
\text { HR }\end{array}$ & $\begin{array}{l}130 / 83 \\
111 / 67 \\
117 / 77 \\
120 / 78 \\
127 / 81 \\
132 / 85 \\
134 / 85 \\
123 / 78 \\
126 / 80 \\
120 / 85 \\
132 / 70 \\
128 / 68\end{array}$ & $\begin{array}{l}91 \\
92 \\
88 \\
98 \\
87 \\
84 \\
85 \\
86 \\
94 \\
99 \\
97 \\
94\end{array}$ & $\begin{array}{l}50 \\
97 \\
90 \\
55 \\
59 \\
52 \\
96 \\
49 \\
40 \\
50 \\
69 \\
72\end{array}$ & $\begin{array}{l}17 \\
18 \\
34 \\
18 \\
17 \\
14 \\
23 \\
11 \\
19 \\
22 \\
12 \\
20\end{array}$ & $\begin{array}{l}33 \\
79 \\
56 \\
37 \\
42 \\
38 \\
73 \\
38 \\
21 \\
28 \\
57 \\
52\end{array}$ & $\begin{array}{r}7.65 \\
6.17 \\
3.44 \\
6.67 \\
7.47 \\
9.43 \\
5.83 \\
11.18 \\
6.63 \\
5.45 \\
11.00 \\
6.40\end{array}$ & $\begin{array}{l}66 \\
81 \\
62 \\
68 \\
71 \\
73 \\
76 \\
78 \\
53 \\
56 \\
82 \\
72\end{array}$ & $\begin{array}{l}170 / 124 \\
163 / 109 \\
158 / 115 \\
185 / 125 \\
172 / 117 \\
179 / 128 \\
165 / 98 \\
155 / 104 \\
165 / 109 \\
150 / 108 \\
178 / 87 \\
163 / 90\end{array}$ & $\begin{array}{l}96 \\
93 \\
95 \\
94 \\
89 \\
84 \\
79 \\
86 \\
92 \\
96 \\
95 \\
98\end{array}$ & $\begin{array}{r}82 \\
103 \\
105 \\
88 \\
80 \\
85 \\
101 \\
65 \\
65 \\
60 \\
102 \\
94\end{array}$ & $\begin{array}{l}31 \\
29 \\
47 \\
43 \\
25 \\
24 \\
33 \\
18 \\
30 \\
30 \\
25 \\
28\end{array}$ & $\begin{array}{l}51 \\
74 \\
58 \\
45 \\
55 \\
61 \\
68 \\
47 \\
35 \\
30 \\
77 \\
53\end{array}$ & $\begin{array}{l}5.48 \\
5.62 \\
3.36 \\
4.30 \\
6.88 \\
7.46 \\
5.00 \\
8.61 \\
5.50 \\
5.33 \\
7.12 \\
5.82\end{array}$ & $\begin{array}{l}62 \\
72 \\
55 \\
49 \\
69 \\
72 \\
67 \\
72 \\
53 \\
50 \\
75 \\
66\end{array}$ \\
\hline $\begin{array}{l}\text { Mean } \\
\text { SD }\end{array}$ & $\begin{array}{l}125 \star / 78^{\star} \\
\pm 7 / 7\end{array}$ & $\begin{array}{l}91^{\star \star} \\
\pm 5\end{array}$ & $\begin{array}{l}65^{\star} \\
\pm 20\end{array}$ & $\begin{array}{l}19 \star \star \\
\pm 6\end{array}$ & $\begin{array}{l}46 \\
\pm 18\end{array}$ & $\begin{array}{l}7 \cdot 28 \star \star \\
\pm 2 \cdot 27\end{array}$ & $\begin{array}{l}70 \\
\pm 9\end{array}$ & $\begin{array}{l}167^{\star \star} / 110^{\star \star} \\
\pm 10 / 13\end{array}$ & $\begin{array}{l}91 \\
\pm 6\end{array}$ & $\begin{array}{l}86^{\star \star} \\
\pm 16\end{array}$ & $\begin{array}{l}30 \star \star \\
\pm 8\end{array}$ & $\begin{array}{l}55^{\star} \\
\pm 14\end{array}$ & $\begin{array}{l}5.87^{\star \star} \\
\pm 1.4\end{array}$ & $\begin{array}{l}64^{\star \star} \\
\pm 9\end{array}$ \\
\hline
\end{tabular}

For key to abbreviations see Table 2. 
Table 4 Haemodynamic data in coronary disease patients (left) 10 minutes after $1 \mathrm{mg}$ atropine given intravenously and (right) during maximum rate of infusion of phenylephrine

\begin{tabular}{|c|c|c|c|c|c|c|c|c|c|c|c|c|c|c|}
\hline \multicolumn{8}{|c|}{ After atropine } & \multicolumn{7}{|c|}{ During maximum phenylephrine infusion } \\
\hline Subject & $\begin{array}{l}B P \\
(m m H g)\end{array}$ & $\begin{array}{l}H R \\
(b p m)\end{array}$ & $\begin{array}{l}E D V I \\
\left(\mathrm{ml} / \mathrm{m}^{2}\right)\end{array}$ & $\begin{array}{l}E S V I \\
\left(m l / m^{2}\right)\end{array}$ & $\begin{array}{l}S V I \\
\left(m l / m^{2}\right)\end{array}$ & $\begin{array}{l}P / V \\
(m m H g / m l \\
\left.\text { per } m^{2}\right)\end{array}$ & $\begin{array}{l}E F \\
(\%)\end{array}$ & $\begin{array}{l}B P \\
(m m H g)\end{array}$ & $\begin{array}{l}H R \\
(b p m)\end{array}$ & $\begin{array}{l}E D V I \\
\left(m l / m^{2}\right)\end{array}$ & $\begin{array}{l}E S V I \\
\left(m l / m^{2}\right)\end{array}$ & $\begin{array}{l}S V I \\
\left(m l / m^{2}\right)\end{array}$ & $\begin{array}{l}P / V \\
(m m H g / m l \\
\left.\text { per } m^{2}\right)\end{array}$ & $\begin{array}{l}E F \\
(\%)\end{array}$ \\
\hline $\begin{array}{l}\text { HC } \\
\text { KB } \\
\text { FC } \\
\text { RS } \\
\text { CD } \\
\text { MW } \\
\text { AH } \\
\text { MR } \\
\text { LG } \\
\text { WW } \\
\text { GM } \\
\text { HP }\end{array}$ & $\begin{array}{l}104 / 75 \\
116 / 70 \\
110 / 80 \\
104 / 76 \\
123 / 65 \\
124 / 101 \\
140 / 92 \\
109 / 53 \\
140 / 80 \\
154 / 90 \\
126 / 86 \\
116 / 80\end{array}$ & $\begin{array}{r}82 \\
96 \\
75 \\
79 \\
101 \\
85 \\
82 \\
93 \\
81 \\
76 \\
77 \\
108\end{array}$ & $\begin{array}{r}75 \\
66 \\
74 \\
128 \\
45 \\
55 \\
55 \\
85 \\
61 \\
128 \\
59 \\
130\end{array}$ & $\begin{array}{r}45 \\
29 \\
41 \\
104 \\
32 \\
28 \\
36 \\
54 \\
21 \\
65 \\
25 \\
101\end{array}$ & $\begin{array}{l}30 \\
37 \\
33 \\
24 \\
13 \\
27 \\
19 \\
31 \\
40 \\
63 \\
34 \\
29\end{array}$ & $\begin{array}{l}2.31 \\
4.00 \\
2.68 \\
1.00 \\
3.84 \\
4.42 \\
3.89 \\
2.02 \\
6.67 \\
2.37 \\
5.04 \\
1.15\end{array}$ & $\begin{array}{l}40 \\
56 \\
45 \\
19 \\
29 \\
49 \\
35 \\
36 \\
65 \\
50 \\
58 \\
22\end{array}$ & $\begin{array}{l}145 / 100 \\
157 / 91 \\
180 / 130 \\
137 / 95 \\
143 / 100 \\
165 / 110 \\
190 / 110 \\
161 / 93 \\
160 / 108 \\
185 / 106 \\
160 / 105 \\
150 / 110\end{array}$ & $\begin{array}{r}86 \\
93 \\
80 \\
81 \\
99 \\
80 \\
79 \\
91 \\
76 \\
72 \\
75 \\
106\end{array}$ & $\begin{array}{r}93 \\
79 \\
146 \\
160 \\
48 \\
76 \\
81 \\
108 \\
79 \\
137 \\
64 \\
151\end{array}$ & $\begin{array}{r}61 \\
47 \\
86 \\
118 \\
40 \\
42 \\
54 \\
75 \\
27 \\
77 \\
35 \\
121\end{array}$ & $\begin{array}{r}32 \\
32 \\
60 \\
42 \\
8 \\
34 \\
27 \\
33 \\
52 \\
60 \\
29 \\
30\end{array}$ & $\begin{array}{l}2.38 \\
3.34 \\
2.09 \\
1.16 \\
3.57 \\
3.93 \\
3.52 \\
2.15 \\
5.93 \\
2.40 \\
4.57 \\
1.24\end{array}$ & $\begin{array}{l}35 \\
41 \\
41 \\
26 \\
17 \\
45 \\
32 \\
30 \\
66 \\
44 \\
45 \\
20\end{array}$ \\
\hline $\begin{array}{l}\mathrm{M} \\
\mathrm{SD}\end{array}$ & $\begin{array}{l}122 / 79 \\
\pm 16 / 13\end{array}$ & $\begin{array}{l}86^{\star \star} \\
\pm 11\end{array}$ & $\begin{array}{c}80 \\
\pm 31\end{array}$ & $\begin{array}{c}48 \\
\pm 28\end{array}$ & $\begin{array}{l}32^{\star} \\
\pm 12\end{array}$ & $\begin{array}{l}3.28 \\
\pm 1.66\end{array}$ & $\begin{array}{l}42 \\
\pm 14\end{array}$ & $\begin{array}{l}161^{\star \star} / 105^{\star \star} \\
\pm 17 / 10\end{array}$ & $\begin{array}{c}85 \\
\pm 10\end{array}$ & $\begin{array}{l}102^{\star \star} \\
\pm 38\end{array}$ & $\begin{array}{l}65^{\star \star} \\
\pm 31\end{array}$ & $\begin{array}{l}37 \\
\pm 15\end{array}$ & $\begin{array}{l}3.02 \\
\pm 1.39\end{array}$ & $\begin{array}{l}37 \\
\pm 13\end{array}$ \\
\hline
\end{tabular}

For key to abbreviations see Table 2.

significant in the normal subjects (from $70 \pm 9$ to $64 \pm 9 \%, \mathrm{p}<0.01)$. Thus, the $\mathrm{P} / \mathrm{V}$ ratio and the ejection fraction appeared to be similarly afterloaddependent in both groups studied.

(2) LINEARITY AND SLOPE OF PEAK SYSTOLIC PRESSURE/END-SYSTOLIC VOLUME RELATION The relation between peak systolic pressure and endsystolic volume was found to be linear over a physiological range of blood pressure $(r \geqslant 0.91)$ in all

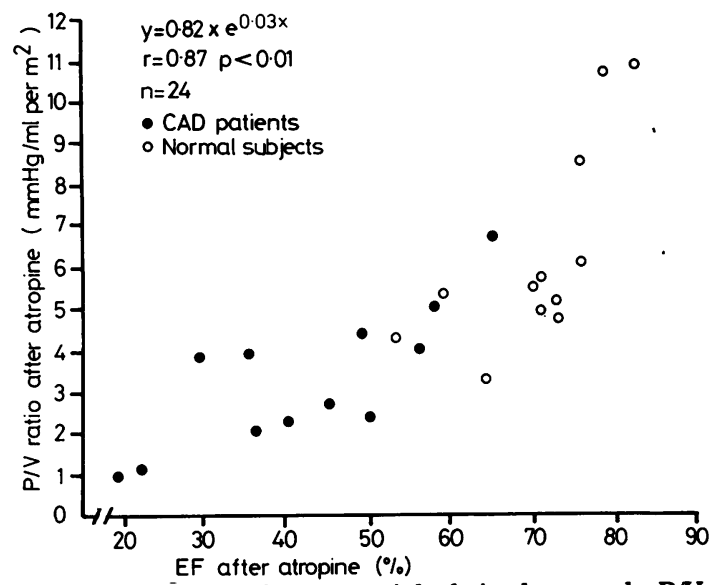

Fig. 2 Plot showing the exponential relation between the $P / V$ ratio and the ejection fraction $(E F)$ determined simultaneously (in this case after atropine).
24 subjects studied. The slopes of these lines were significantly steeper in normal subjects than in the patients with coronary artery disease (3.9 \pm 0.9 $\mathrm{mmHg} / \mathrm{ml}$ per $\mathrm{m}^{2}$ compared with $2.5 \pm 0.6 \mathrm{mmHg} / \mathrm{ml}$ per $\left.\mathrm{m}^{2}, \mathrm{p}<0.05\right)$ though there was some overlap of individual values between the two groups (Fig. 3). Ten of 12 normal subjects, however, but only four of 12 patients with coronary disease had a $\mathrm{P} / \mathrm{V}$ slope $>2.7 \mathrm{mmHg} / \mathrm{ml}$ per $\mathrm{m}^{2}$.

The P/V slope correlated exponentially with the ejection fraction determined at the same heart rate (that is after atropine), as shown in Fig. 4. Thus, as with the $\mathrm{P} / \mathrm{V}$ ratio, most of the normal subjects fell on the steep part of the curve, where the ejection fraction is relatively insensitive to changes in the $P / V$ slope.

The observed intercept of the P/V slope on the volume axis was not significantly different between the two groups (see Table 5 for individual linear regression data).

(3) THEORETICAL VALUE FOR END-SYSTOLIC VOLUME AT ZERO SYSTOLIC PRESSURE-V $\mathrm{V}_{0}$ was calculated as $-b / a$ where $a$ is the $\mathrm{P} / \mathrm{V}$ slope and $b$ is the observed intercept on the volume axis. Mean $V_{0}$ was $-16 \pm 10 \mathrm{ml} / \mathrm{m}^{2}$ in the normal subjects and $-4 \pm 25 \mathrm{ml} / \mathrm{m}^{2}$ in the coronary artery disease group (NS). No significant correlation was found between $V_{0}$ and ejection fraction overall, though there appeared to be a trend towards an inverse relation with values of less than $50 \%$ for ejection fraction (Fig. 5). 


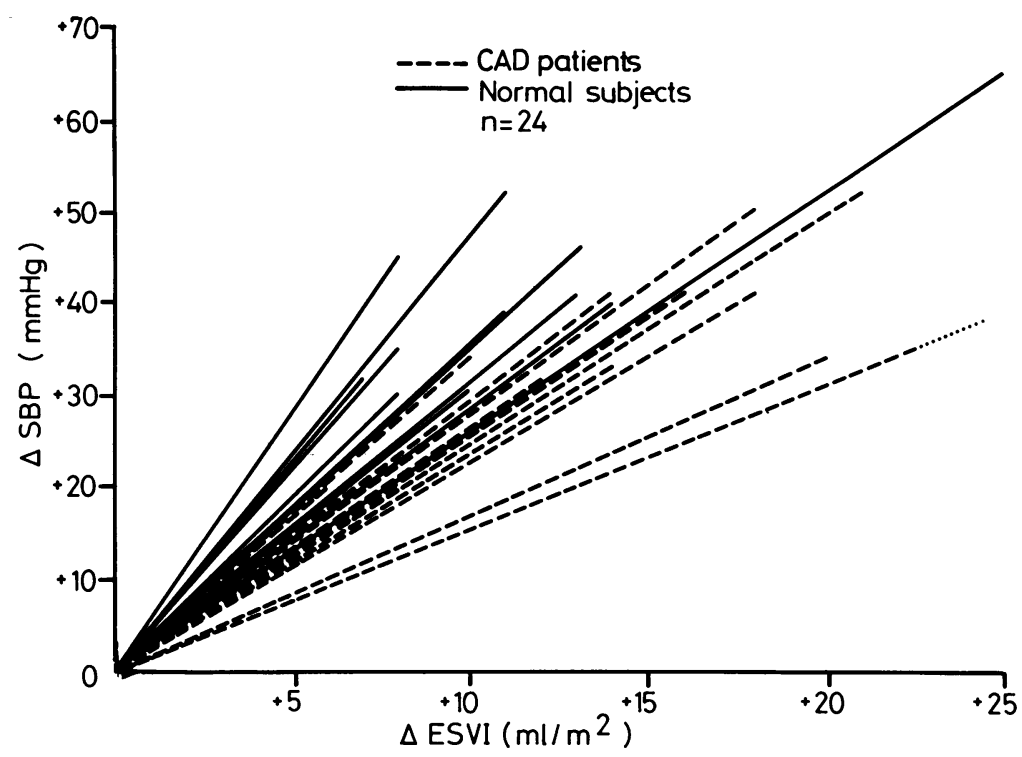

Fig. 3 Individual plots showing the linear relation between the increase in systolic blood pressure and the resultant rise in end-systolic volume. The slope of these lines in the patients with coronary artery disease tends to be less steep than in normal subjects, but there is some overlap of the two groups.

Table 5 Characteristics of peak systolic pressurelend-systolic volume relation over physiological range of systolic blood pressure

\begin{tabular}{|c|c|c|c|c|c|c|c|c|c|}
\hline \multicolumn{5}{|c|}{ Normal subjects } & \multicolumn{5}{|c|}{ Coronary disease patients } \\
\hline Subject & $r$ & Slope & Intercept & $V_{0}$ & Subject & $r$ & Slope & Intercept & $V_{0}$ \\
\hline $\begin{array}{l}\text { WC } \\
\text { DJ } \\
\text { ML } \\
\text { RC } \\
\text { MB } \\
\text { GM } \\
\text { PW } \\
\text { DE } \\
\text { WE } \\
\text { WM } \\
\text { SS } \\
\text { HR }\end{array}$ & $\begin{array}{l}0.97 \\
0.98 \\
0.99 \\
0.99 \\
0.98 \\
0.95 \\
0.99 \\
0.99 \\
0.91 \\
0.99 \\
0.96 \\
0.97\end{array}$ & $\begin{array}{l}2.7 \\
5.0 \\
3.3 \\
2.6 \\
5.2 \\
4.8 \\
3.1 \\
4.6 \\
3.3 \\
3.8 \\
3.5 \\
4.4\end{array}$ & $\begin{array}{r}+88 \\
+19 \\
+6 \\
+73 \\
+39 \\
+68 \\
+63 \\
+72 \\
+53 \\
+40 \\
+96 \\
+45\end{array}$ & $\begin{array}{r}-33 \\
-4 \\
-2 \\
-28 \\
-8 \\
-14 \\
-20 \\
-16 \\
-16 \\
-11 \\
-27 \\
-10\end{array}$ & $\begin{array}{l}\text { HC } \\
\text { KB } \\
\text { FC } \\
\text { RS } \\
\text { CD } \\
\text { MW } \\
\text { AH } \\
\text { MR } \\
\text { LG } \\
\text { WW } \\
\text { GM } \\
\text { HP }\end{array}$ & $\begin{array}{l}0.98 \\
0.93 \\
0.99 \\
0.93 \\
0.96 \\
0.94 \\
0.99 \\
0.97 \\
0.98 \\
0.99 \\
0.99 \\
0.98\end{array}$ & $\begin{array}{l}2.5 \\
2.8 \\
1.6 \\
2.1 \\
2.0 \\
2.7 \\
2.6 \\
2.5 \\
3.8 \\
2.8 \\
3.3 \\
1.6\end{array}$ & $\begin{array}{r}-7 \\
+63 \\
+46 \\
-105 \\
+59 \\
+57 \\
+50 \\
-28 \\
+61 \\
-25 \\
+36 \\
-51\end{array}$ & $\begin{array}{r}+3 \\
-23 \\
-29 \\
+50 \\
-30 \\
-21 \\
-20 \\
+11 \\
-16 \\
+9 \\
-11 \\
+32\end{array}$ \\
\hline $\begin{array}{l}M \\
S D\end{array}$ & $\begin{array}{r}0.97 \\
\pm 0.02\end{array}$ & $\begin{array}{r}3.9 \\
\pm 0.9\end{array}$ & $\begin{array}{l}+55 \\
\pm 27\end{array}$ & $\begin{array}{l}-16 \\
\pm 10\end{array}$ & & $\begin{array}{r}0.97 \\
\pm 0.02\end{array}$ & $\begin{aligned} & 2 \cdot 5^{\star} \\
\pm & 0.6\end{aligned}$ & $\begin{array}{r}13 \\
\pm 55\end{array}$ & $\begin{array}{r}-4 \\
\pm 25\end{array}$ \\
\hline
\end{tabular}

${ }^{\star} \mathrm{p}<0.01$ compared with normal subjects. $r$, linear coefficient of correlation between systolic BP and end-systolic volume over a physiological range of $B P$, that is $y \mathrm{mmHg}=a x+b \mathrm{ml} / \mathrm{m}^{2}$ where $a=$ slope, $b=$ intercept, and $V_{0}=-b / a$ (theoretical end-systolic volume index at zero pressure).

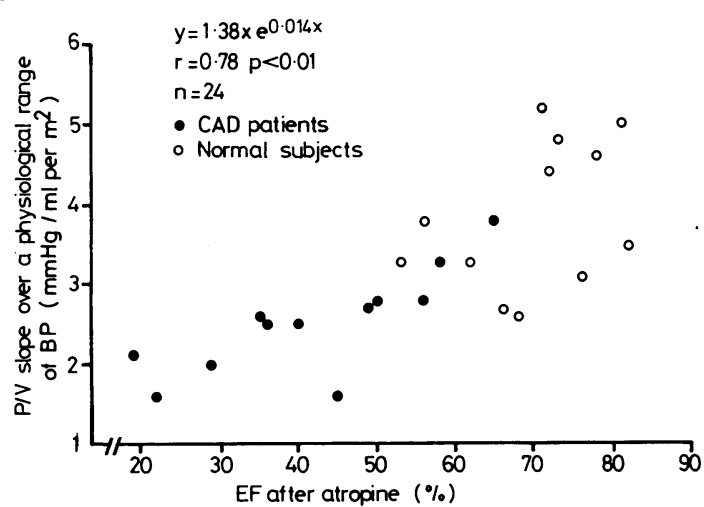

Fig. 4 Plot showing the exponential relation between the $P / N$ slopes seen in Fig. 3 and the ejection fraction determined at the same heart rate, that is immediately after atropine.

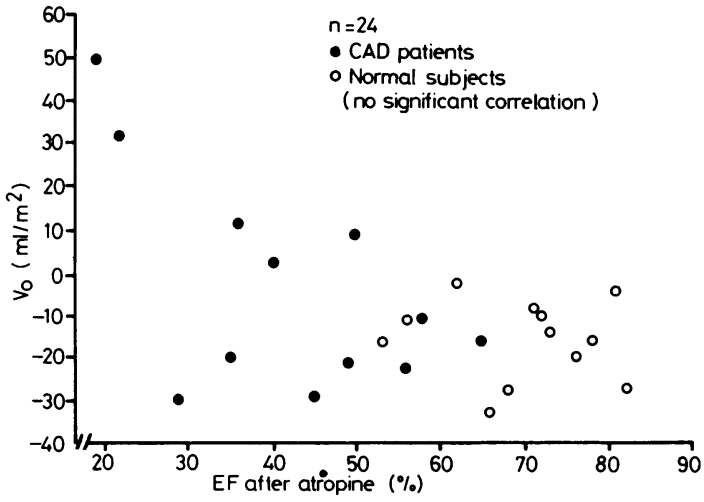

Fig. 5 Plot of $V_{0}$ against ejection fraction (EF) determined at the same heart rate. Though no significant correlation was found overall, there was a trend towards an inverse correlation in patients whose post-atropine ejection fraction $(E F)$ was $<36 \%$. 


\section{Discussion}

This study has confirmed the linearity of the peak systolic pressure/end-systolic volume relation in man at a constant inotropic state and over a physiological range of blood pressure. Previous studies using $M$-mode echocardiography ${ }^{16}$ and contrast ventriculography ${ }^{6}$ have also shown a linear relation but, unlike radionuclide ventriculography, both these methods are dependent on geometric assumptions about the shape of the left ventricle at end-systole, assumptions that may be particularly inappropriate in patients with previous myocardial infarction.

The slope of this relation has been shown in previous clinical and experimental studies to be an index of inotropic state. 2416

We found an exponential relation between the P/V slope and the ejection fraction, confirming the findings of Mehmel et al. ${ }^{6}$ Most normal subjects fell on the steep part of the curve (see Fig. 4) suggesting that the P/V slope might be more sensitive to changes in inotropic state than the ejection fraction, at least in such normal subjects.

Although the mean P/V slope was steeper in the normal subjects than in those with coronary disease, there was considerable overlap between the two groups, presumably because the resting contractile state of the left ventricle is not invariably depressed after myocardial infarction.

The ejection fraction is commonly used in clinical practice as a measure of the contractile state of the left ventricle, but it is also influenced by changes in heart rate, preload, and afterloading conditions. Thus, when preload is decreased or afterload increased, the ejection fraction is depressed even if the inotropic state of the left ventricle is constant. Despite this limitation, the ejection fraction is widely used to detect, quantify, and prognosticate upon left ventricular dysfunction in patients with coronary artery disease..$^{17-19}$

The P/V slope, however, being linearly afterload dependent and virtually unaffected by preload, may more accurately reflect the inotropic state of the left ventricle than the ejection fraction under circumstances where both cardiac loading conditions and inotropic state are changing. Indeed, the $\mathrm{P} / \mathrm{V}$ relation is now being used to evaluate cardiovascular drug effects in man. 1020

By contrast, the extrapolated value of end-systolic volume at zero pressure $\left(V_{0}\right)$ did not correlate significantly with the ejection fraction in our study, nor was it significantly different in subjects with and without left ventricular dysfunction caused by coronary artery disease. This is in contrast to the findings of Grossman et al., ${ }^{4}$ who observed a significantly higher value for $V_{0}$ in patients with a depressed ejection fraction than those with a normal ejection frac- tion, as assessed by contrast ventriculography.

Only the subgroup of five patients with severely impaired myocardial performance in Grossman's study (mean ejection fraction $24 \%$ ), however, had a significantly higher value of $V_{0}$, and this tendency was observed in the five patients in our study with an ejection fraction $<36 \%$ (see Fig. 5). In addition, there were no normal subjects in Grossman's study, and those with normal ejection fractions had evidence of systolic volume overload (mean end-systolic volume was $51 \mathrm{ml} / \mathrm{m}^{2}$ ). Thus, it appears that $V_{0}$ is not a sensitive index of myocardial performance in normal subjects and seems to be consistently raised only when both myocardial contractility is severely impaired and end-systolic volume is greatly enlarged.

The P/V ratio was of similar sensitivity to the ejection fraction in the detection of patients with left ventricular dysfunction caused by coronary artery disease, at rest and after each of the pharmacological interventions. One interesting difference was in the response to atropine in the normal subjects. The $P / V$ ratio rose in all 12 subjects after atropine, whereas the ejection fraction only rose in four subjects. Since it is now established that an increase in heart rate per se constitutes a positive inotropic stimulus in man, ${ }^{21}$ these findings further support the increased sensitivity of the systolic $P / V$ relation over the ejection fraction as an index of the prevailing inotropic state.

Increasing afterload on the left ventricle with phenylephrine led to a similar dose-dependent decline in both the P/V ratio and the ejection fraction-both more pronounced in the normal subjects than in the patients with coronary artery disease. It was assumed for the purposes of this study that phenylephrine infusion in the presence of an atropine-induced vagolysis had no reflex effects upon the inotropic state of the left ventricle. No patient complained of chest pain or had electrocardiographic abnormalities during the phenylephrine infusion, but nearly all subjects had a transient throbbing headache at the highest dose.

- Isometric and dynamic exercise generally increase the sensitivity of indices of myocardial performance for differentiating normal subjects from those with left ventricular dysfunction caused by coronary artery disease. This has proved to be the case with studies using the systolic pressure/volume relation. ${ }^{78}$ The response of both blood pressure and heart rate to any given level of exercise, however, may vary widely, both within and between subjects. We felt that pharmacological afterload stress, with control of the systolic blood pressure and heart rate, might further increase the sensitivity of this index for detecting minor degrees of left ventricular dysfunction caused by coronary artery disease. The fact that no such increase in sensitivity was seen may be because our 
study did not incorporate a positive inotropic stimulus such as that which occurs with exercise, and a study using isoprenaline under similar conditions is in progress.

One potential limitation on the interpretation of our findings is the younger mean age of the normal subjects. We are not aware of any studies that show an effect of age per se on ejection phase indices of myocardial contractility. Aortic impedance may increase with age and it is thus possible that this would further depress the ejection fraction and the $\mathrm{P} / \mathrm{V}$ ratio in the coronary disease group. ${ }^{22}$

In conclusion, this study has evaluated the clinical utility of the peak systolic pressure end-systolic volume relation in normal subjects and patients with coronary artery disease. While neither the $P / V$ ratio nor the P/V slope appeared to be significantly better than the ejection fraction in the detection of left ventricular dysfunction resulting from coronary artery disease, the P/V ratio may more closely reflect the prevailing inotropic state of the left ventricle. Unlike both the P/V ratio and the ejection fraction, the P/V slope is independent of afterload and may therefore have special utility in the evaluation of cardiovascular drug effects in man.

\section{References}

1 Suga H, Sagawa K, Shoukas AA. Load independence of the instantaneous pressure-volume ratio of the canine left ventricle and effects of epinephrine and heart rate on the ratio. Circ Res 1972; 32: 314-22.

2 Suga H, Sagawa K, Kostiuk DP. Control of ventricular contractility assessed by the pressure-volume ratio, E max. Cardiovasc Res 1976; 10: 582-92.

3 Mahler F, Covell JW, Ross J Jr. Systolic pressurediameter relations in the normal conscious dog. Cardiovasc Res 1975; 9: 447-55.

4 Grossman W, Braunwald, E, Mann T, McLaurin LP, Green LH. Contractile state of the left ventricle in man as evaluated from end-systolic pressure-volume relations. Circulation 1977; 56: 845-52.

5 Nivatpumin T, Katz S, Scheuer J. Peak left ventricular systolic pressure/end-systolic volume ratio: a sensitive detector of left ventricular disease. Am $\mathcal{F}$ Cardiol 1979; 43: 969-74.

6 Mehmel HC, Stockins B, Ruffmann K, Ick A, Kübler $W$. The endsystolic pressure-volume relation in man: its linearity and sensitivity for the assessment of left ventricular function (abstract). Circulation 1979; 60, suppl 2: 121.

7 Slutsky R, Karliner J, Gerber K, et al. Peak systolic blood pressure/end systolic volume: assessment at rest and during exercise in normal subjects and patients with coronary heart disease. Am $\mathcal{F}$ Cardiol 1980; 46: 813-20.

8 Dehmer GJ, Lewis SC, Corbett J, Hillis LD, Parkey $R W$, Willerson JT. The end-systolic P/V index: a new parameter for the scintigraphic evaluation of coronary artery disease. Clin Res 1980; 28: 165A.

9 Slutsky R, Watkins J, Peterson K, Karliner J. The response of left ventricular function and size to atrial pacing, volume loading and afterload stress in patients with coronary artery disease. Circulation 1981; 63: 864 70.

10 Merillon JP, Motte G, Aumont MC, Prasquier R, Gourgon $R$. Study of left ventricular pressure-volume relations during nitroprusside infusion in human subjects without coronary artery disease. $\mathrm{Br}$ Heart $\mathrm{F}$ 1979; 41: 325-30.

11 Smith TD, Richards P. A simple kit for the preparation of ${ }^{99 m}$ Tc labeled red blood cells. $\mathcal{F}$ Nucl Med 1976; 17: 126-32.

12 Slutsky R, Battler A, Gerber K, et al. A simplified method for the calculation of left ventricular volume by equilibrium radionuclide angiography. Cathet Cardiovasc Diagn 1980; 6: 49-60.

13 Slutsky R, Karliner J, Ricci D, et al. Left ventricular volumes by gated equilibrium radionuclide angiography: a new method. Circulation 1979; 60: 556-64.

14 Slutsky R, Karliner JS, Battler A, Pfisterer M, Swanson $S$, Ashburn W. Reproducibility of ejection fraction and ventricular volume by gated radionuclide angiography after myocardial infarction. Radiology 1979; 132: 155-9.

15 Slutsky R, Pfisterer M, Verba J, Battler A, Ashburn W. Influence of different background and left ventricular assignments on ejection fraction results. Radiology 1980; 135: 725-30.

16 Marsh JD, Green LH, Wynne JH, Cohn PF, Grossman W. Left ventricular end systolic pressure-dimension and stress-length relations in normal human subjects. $A m \mathcal{F}$ Cardiol 1979; 44: 1311-7.

17 Nelson GR, Cohn PF, Gorlin R. Prognosis in medically treated coronary artery disease. Influence of ejection fraction compared with other parameters. Circulation 1975; 52: 408-12.

18 Gorlin R. Evaluation of patients with coronary artery disease. In: Gorlin R, ed. Coronary artery disease. Philadelphia: WB Saunders, 1976.

19 Maddox DE, Holman BL, Wynne J, et al. Ejection fraction image: a noninvasive index of regional left ventricular wall motion. Am $\mathcal{f}$ Cardiol 1978; 41: 1230-8.

20 Slutsky R, Watkins J, Costello D. The systolic pressure/ end systolic volume relationship: response to pharmacologic agents in patients with coronary heart disease. $A m$ Heart $\mathcal{F}$ (in the press).

21 Ricci DR, Orlick AE, Alderman EL, et al. Role of tachycardia as an inotropic stimulus in man. $\mathcal{F}$ Clin Invest 1979; 63: 695-703.

22 Milnor, WR. Arterial impedance as ventricular afterload. Circ Res 1975; 36: 565-70.

Requests for reprints to Dr J Watkins, Department of Cardiovascular Medicine, John Radcliffe Hospital, Headington, Oxford OX3 9DU. 